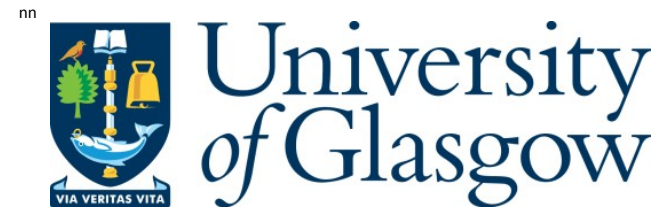

U roukov, I., and Speller, R. (2015) A preliminary approach to intelligent Xray imaging for baggage inspection at airports. Signal Processing Research, 4 . pp. 1-11. ISSN 2327-1701

Copyright $\Subset 2015$ The A uthors

http://eprints.gla.ac.uk/101423/

Deposited on: 16 J anuary 2015

Enlighten - R esearch publications by members of the U niversity of Glasgow http://eprints.gla.ac.uk 


\title{
A Preliminary Approach to Intelligent X-ray Imaging for Baggage Inspection at Airports
}

Application to the detection of threat materials and objects

\author{
Ivan S. Uroukov ${ }^{*}$ and Robert Speller ${ }^{2}$ \\ 1-MRC - University of Glasgow Centre for Virus Research.8 Church Str., Glasgow, G11 5JR., Scotland, UK. \\ 2-Department of Medical Physics and Bioengineering,Malet Place Engineering Building,University College \\ London, Gower Street, London, U.K.WC1E 6BT. \\ * Corespondent author. He is a former member of R. Speller's group at UCL. \\ *1ivan.uroukov@glasgow.ac.uk; ${ }^{2}$ r.speller@ucl.ac.uk
}

\begin{abstract}
Identifying explosives in baggage at airports relies on being able to characterize the materials that make up an X-ray image. If a suspicion is generated during the imaging process (step 1), the image data could be enhanced by adapting the scanning parameters (step 2).

This paper addresses the first part of this problem and uses textural signatures to recognize and characterize materials and hence enabling system control. Directional Gabor-type filtering was applied to a series of different $\mathrm{X}$-ray images. Images were processed in such a way as to simulate a line scanning geometry. Based on our experiments with images of industrial standards and our own samples it was found that different materials could be characterized in terms of the frequency range and orientation of the filters. It was also found that the signal strength generated by the filters could be used as an indicator of visibility and optimum imaging conditions predicted.
\end{abstract}

\section{Keywords}

X-ray Imaging and Vision; X-ray Image Based Material Discrimination; Gabor Filters; Intelligent Adaptive Imaging; Energetic Materials; Liquid and Plastic Explosives; Weapons; Material Discrimination

\section{Introduction}

Baggage carried on board aircraft or post check-in baggage is subject to X-ray inspection to identify illegal materials such as weapons or explosives. To enhance the detection of these threats it has been proposed that intelligent baggage scanning based on the I-ImaS concept (Griffiths et al. 2008) could be used. The outcome would be a personalized inspection, tailored to each piece of baggage, meaning an enhanced level of threat detection. The I-ImaS process requires a dual line scanning approach where the first line scan generates data that could be used to intelligently adapt the scanning parameters for the second line scan data collection. Such a concept has shown to enhance detection in medical imaging scenarios (Martin et al. 1999, Huda et al. 2003) and could be used to enhance the performance of current inspection techniques. Current levels of false positives/false negatives achieved in airport security systems is a closely guarded secret but in any X-ray imaging application optimization of imaging parameters will lead to image quality improvements. Unlike medical imaging systems where exposure factors can be modified on a patient by patient basis this has not been feasible in airport security systems as it would require operator intervention for every piece of scanned baggage. Using the I-ImaS concept this optimization process would be happen automatically ensuring that the highest quality, most informative information is presented to the operator irrespective of the type, size and density of the objects being scanned. Hence the X-ray baggage scanners of the future would provide 'personalised' inspection and hence mirror the aspirations of the medical profession.

Enhancement of baggage scanning images has been studied using image processing techniques postacquisition of the image by several authors. The essential processing steps (Woodell G. et al. 2004) have been histogram modifications, high-pass filtering, sharp (\&/or) unsharp masking or other auto-level methods on the gray scale images such as scene decluttering (Abidi B. at al. 2004). However, none of these techniques have been proposed as feedback signals to enhance the acquisition of image data.

Novel combined hardware and software methods such as software-filtering and (Zhiyu Chen et al. 2005.) dual energy acquisition techniques have been 
developed but these do not use any real-time adaptation to optimize detection. Shape based techniques were developed to detect concealed weapons (Franzel T., et al. 2012, Gesick R, et al. 2009) including multiple view $\mathrm{x}$-ray techniques (Merry D et al. 2013) but, generally these are limited to specific threat shapes.

More success was demonstrated but with significant computational and hardware costs by using a combination of multi energy computed tomography and object recognition techniques (Willson P. D. et al. 1997). Here automated object detection is significantly dependent on predefined shape and material targets.

In medical work the use of adaptive imaging has generally, but not exclusively, been used to optimize the dose to the patient. Adaptive frame scanning with pulsed X-rays for control and minimizing the radiation dose was developed by Farhad Ghelmansarai, Perkin Elmer Patent 2012andthe I-ImaS approach has been shown to reduce dose by up to $60 \%$ in comparison to conventional imaging systems.

It is a two-step process (see Longo et al. 2007) which uses adaptive analysis of the images to create maximum diagnostic information within given dose constraints as shown by Griffiths et al. 2008. Firstly a limited area is imaged and based upon analysis of that data the second step involves change or no-change to the imaging parameters for the second 'look' at that area.

Adaptive or intelligent baggage scanning is also a two step process that must be undertaken on a strip by strip process as the bag passes through the baggage scanner. If the beam from the X-ray source is divided into two separate line scans then as the bag passes through the system it will encounter two fan beams of radiation. The encounter with the first beam allows step one in the two step process to take place followed by step two when the bag reaches the second beam. In the first step enough information needs to be gathered about a strip within the bag for a decision to be made about the contents. If the contents are suspicious then the imaging conditions at step two need to be adjusted to allow a more informed decision to be made. Unlike the medical case where changes are made to optimize dose in the security example changes would be made to enhance the detection of suspicious regions. The changes made to the imaging conditions could include altering the $\mathrm{kV}$ or $\mathrm{mA}$ on the $X$-ray tube, using different $X$-ray filters, increasing or deceasing the pixel size of the detector by binning or unbinning pixels, or slowing down the belt speed to allow more X-ray photons to be detected.

The work reported in this paper deals with step one and develops a novel technique for deciding whether or not a region can be classified as suspicious an hence invoke some form of change to the imaging parameters. Understanding what form that change should take is currently being researched and will be reported at a later date.

Making decisions on local regions is central to the operation of such a systemand the decision based on textural analysis of the acquired image has shown successful results in the medical field ( A.R. ALHinnawi and M. Daear, 2012). The current paper concentrates on a novel technique, based on textural information to aid in that decision in the context of security scanning. In particular the technique simulates how a human observer would respond to the image data to carry out the optimization of imaging parameters.

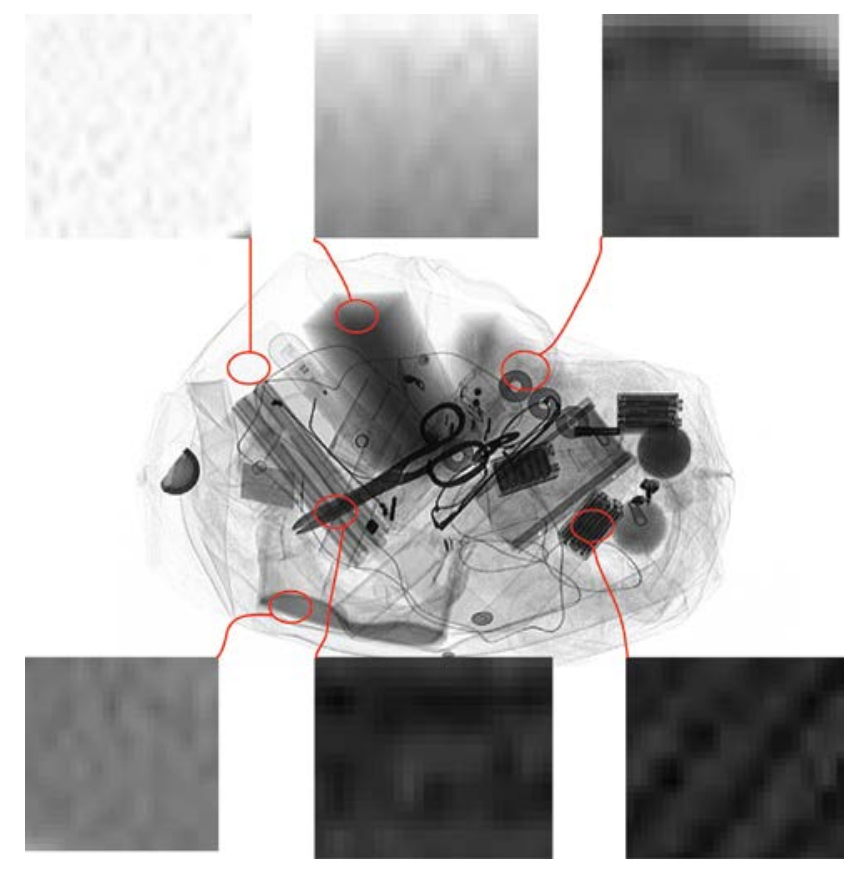

FIG. 1 ILLUSTRATION OF TEXTURE EXAMPLES FROM A REAL BAGGAGE SCAN INCLUDING OBJECTS FROM TEXTILES, METALS, ORGANIC AND COMBINATION OF THEM.

\section{Human Factors in Image Interpretation}

Texture is an important characteristic of X-ray imaged materials in passenger's luggage. Here we implement textural analysis of materials based upon the spatial variation of the gray-levels that make up the image.

Figure 1 illustrates examples of texture from a real baggage scan. The examples are from various materials ranging from textiles, metals organic and 
combinations of them. Magnified areas (red lines) from various objects illustrate gray level variation in the region of the object, which forms the texture. Texture is widely employed for feature analysis and, for example, has been implemented in Synthetic Aperture Radar (SAR) analysis on ice (Ulaby F.et al. 1986.), signal classification in radar target recognition (Yu Shi and Xian-Da Zhang 2001) and in malignancy detection (Mohamed S. S et al. 2003). Gray level variation (i.e. texture) is widely used for various applications such as biometrical applications in fingerprint and iris analysis, as well as a combination of them as shown in Yunhong Wang et al., 2003, where textural features are fundamental in generating a map of image features.

The research history on textural analysis in imaging fields (Clausi D.A. and Jernigan M.E , 2000) suggests that simple cells in mammals and the human vision system (HVS) may be modeled by means of textural perception shown by Julesz (Julesz B. 1962, Julesz B.1981, Julesz B.2011). In this work we do not reflect on color and stereopsis perception. The HVS has been found to be sensitive to the frequency, direction and orientation of the image elements. Psychophysical visual experiments by by Rao A.R. and Lohse G.L., 1993, showed high-level features for texture discrimination, such as repetition, directionality and complexity are used by participating human observers. Directionality and repetition are represented by the orientation and frequency whilst complexity is related to texture consistency.

Furthermore, Marcelia S. (1980) and Daugman J. (1985) modeled receptive fields of simple cells in the primary visual cortex of mammals with 2D Gabor functions, yielding indistinguishable residual error with experimental data similar to Jones and Palmer (1987). Other computational models of periodic and aperiodic pattern selective cells, called grating and bar-cells (found in areas V1 and V2 of monkey's visual cortex) were presented by Petkov et al. (1997). A model of a simple cell has been presented by Azzopardi et al 2012 where the authors use Gaussian in favor of Gabor filters. These cells respond strongly to images of bar gratings of a given orientation and frequency but very weakly or not at all to single bars. Campbell and Kulikowski (1966), found that humans have orientation and spatial frequency sensitivity, which can be modeled as a number of independent detector mechanisms; each represented by a narrow band filter tuned to a different frequency. Gabor filters have both frequency and orientation selective properties. They possess optimal joint resolution in the spatial and frequency domain (Daugman J. (1985), Jain A.K. (1991)) and are therefore suited for modeling the receptive field of simple cells in the HVS.

\section{Physical Factors in Image Formation}

An important factor is to relate the object thickness and absorption to its textural content. X-ray transmission of the detected signal depends upon the thickness and type of material, i.e., the radiation pathlength. However, in most baggage scanning examples the total thickness of the object, i.e., the suitcase and its contents, does not change significantly and hence changes in the recorded X-ray transmission, i.e., grey levels in the image, relates primarily to the attenuation coefficient of the materials that make up the suitcase contents.

To address the variation in the content of the luggage and packaging a number of examples were used including:

- industry standards obtained from 3DX-RAY Ltd, Loughborough, UK

- packages constructed following discussion with U.K. Home Office, and

- liquids.

Other factors such as beam hardening will not be a problem as baggage scanners operate with a prehardened beam quality. Scattering is also minimal due to the use of narrow $\mathrm{X}$-ray fan beams.

Thus in the current work we use a Gabor filter bank as a textural 'analyzing core' within an airport security scanning development based on the I-ImaS principle. In doing this we generate a novel feature space of material descriptors ('Uroukov's code') based on the textures of imaged material. This space is used to generate a map of targeted materials that can be used for feedback to the scanning system to enhance the information, i.e., the image, presented to the operator.

\section{Visibility Model and 'Uroukov's Code'}

The textural analysis uses an X-ray grey scale image and is based on 'seeing' textures where textures are associated with object properties more strongly than the mean grey levels. The main difference between the mean grey level and texture metric is that the latter relies on the differences in the spatial arrangement of the grey levels. The intensity of X-rays transmitted through an object is related to $e^{-\mu x}$ where $\mu$ is the attenuation coefficient and $x$ is the thickness of 
material. The grey level recorded by a detector is directly related to the transmitted intensity. However, many materials are undistinguishable even when dual energy techniques are used. Real images demonstrate advanced textural properties and hence, textural characterization is more robust at characterizing materials than just using absorption measures.

\section{'Seeing' Textures and Gabor Filters.}

Biologically inspired image processing (see Marcelja 1980, Daugman 1980,1985) has shown that the human visual system performs in a multi-resolutional decomposition manner. Wavelets are intrinsically multi-resolutional and they have been used to model and detect textures (see Coggins J.M. and Jain A.K., 1985, Jain A. K. at al.1997). In this work the Gabor function (see Daugman 1980) has been used to design a multi-channel filter bank, i.e., a range of filters based upon the Gabor function that vary in frequency

A Gabor function is a Gaussian modulated by a sinusoid. It is written as the product of an elliptical Gaussian (centered at $x, y$ ) and a complex sinusoid :

$$
f(x, y)=\frac{1}{2 \pi \sigma_{x} \sigma_{y}} e^{\left(-\frac{1}{2}\left(\frac{\left(x-X_{c}\right)^{2}}{\sigma_{x}^{2}}+\frac{\left(y-Y_{c}\right)^{2}}{\sigma_{y}^{2}}\right)\right)} e^{\left(2 \pi j\left[U\left(x-X_{c}\right)+V\left(y-Y_{c}\right)\right]\right)}
$$

where:

$\sigma_{x}, \sigma_{y}$ are spatial (space) constants of the Gaussian envelop along $x$ and $y$ axes respectively.

$X_{c}, Y_{c}$ - coordinates at which Gaussian is centered.

$U, V$ - define the harmonic spatial frequency in Cartesian coordinates such as

$F=\sqrt{U^{2}+V^{2}}$ is the magnitude of spatial frequency in polar coordinates and direction $\theta=\tan ^{-1}(V / U)$, where $U=F \cos \theta, V=F \sin \theta$.

The complete filter is made up of even and odd components. In texture research (see Jain and Farroknia1991) it is widely accepted that a bank of real components of a Gabor filter is sufficient to represent the channels, hence:

$$
h(x, y)=\frac{1}{2 \pi \sigma_{x} \sigma_{y}} e^{\left(-\frac{1}{2}\left(\frac{x_{\theta}^{2}}{\left.\left.\sigma_{x}^{2}+\frac{y_{\theta}^{2}}{\sigma_{y}^{2}}\right)\right)} \cos \left(2 \pi f x_{\theta}\right)\right.\right.}
$$

where

$x_{\theta}=x \cos \theta+y \sin \theta, y_{\theta}=-x_{\theta} \sin \theta+y \cos \theta$

$x, y$ denotes coordinates of pixels in the image
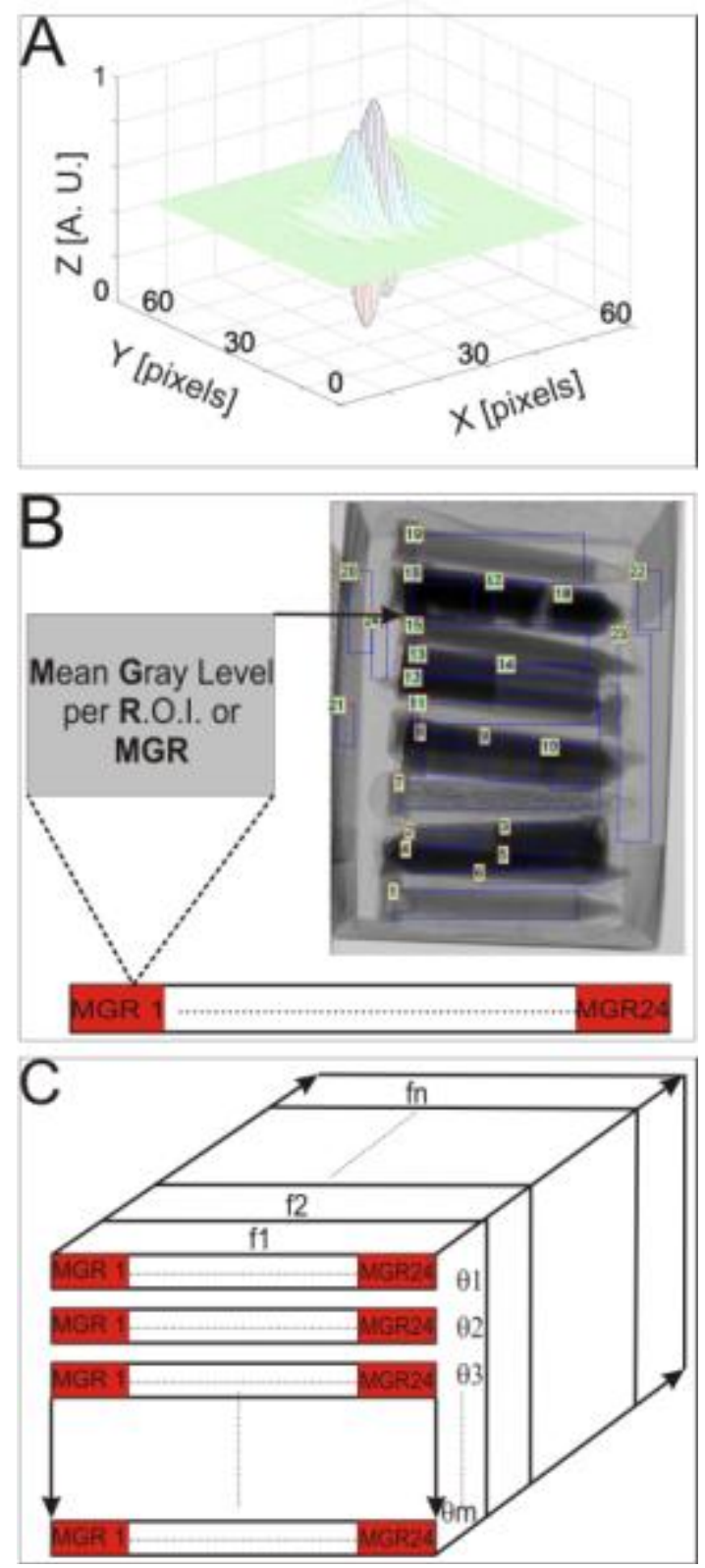

FIG. 2 A FILTER KERNEL DESIGNED WITH A CIRCULAR GAUSSIAN $\sigma_{x}=\sigma_{y}=\sigma=6$ [PIXELS]. THE SIZE OF THE MASK IS 51X51 PIXELS AND THE PERIOD IS $T=6 \sqrt{2}$. THE RADIAL FREQUENCY IS $\varpi=2 \pi / 6 \sqrt{2}=0.74[$ revol. $/$ img.width] AND ORIENTATION IS $\theta=0^{\circ}$.

B FORMATION OF THE FILTER BANK RESPONSE IN UROUKOV'S CODE. FOR RESEARCH PURPOSES A SET OF KNOWN MATERIALS MAY BE USED TO TRAIN THE SYSTEM TO IDENTIFY HAZARDOUS SUBSTANCES OF INTEREST, I.E.

PLASTIC OR LIQUID ENERGETIC MATERIALS. PRACTICALLY, A SET OF LOCALIZED AREAS FROM THE IMAGE ARE SELECTED AND TAGGED BY THEIR COUNTING ORDER, SIGNIFYING THE REGIONS OF INTEREST WHERE THE MATERIALS ARE LOCALIZED. AFTER FILTERING, THE MEAN LEVELS OF PARTICULAR ROIS FORM A VECTOR AT A PARTICULAR FREQUENCY AND ORIENTATION. THIS IS ILLUSTRATED AS MGR1.MGR $n$ AND IS LABELLED THE MGR VECTOR.

C THE ARRANGEMENT OF THE MGR VECTORS OVER A RANGE OF FREQUENCIES AND ORIENTATIONS IN UROUKOV'S CODE. 
$\theta$ is the orientation parameter of the Gabor filter

$f$ is the frequency parameter of a sinusoidal plain wave

An example of the kernel is shown in Figure 2A where the Gaussian is circular. Filtering is a convolution of the image with the mask, where the parameters $\varpi, . \theta$ are set in advance. A decision on the circularity of the Gaussian has been influenced by other work where circular Gaussians were used (Lin Hong et al 1998) along with the selection of a value for $\sigma$ (the standard deviation)which involves a trade-off for a circular Gaussian, where $\sigma=\sigma_{x}=\sigma_{y}$ (Lin Hong et al 1998). The work cited deals with fingerprint analysis where a close link with a circular function is more obvious. In the mixed textures expected within images of suitcase contents it was decided not to give a bias to any preferred textural shapes and hence a circular function was chosen.

The algorithms were developed in the Matlab ${ }^{\circledR}$ (Release 2009), The MathWorks Inc. language and were run on a Dell Precision Workstation with $2 \mathrm{x}$ Intel ${ }^{\circledR}$ Xeon ${ }^{\circledR}$ Quad Core processors with 12 GB of RAM. Typical run times were les then a second, which could be significantly influenced, if implemented on dedicated hardware platform. These algorithms have also been successfully used in a different application (Uroukov, et al. 2014).

\section{Understanding the Textural Information.}

To understand the efficiency of detecting different materials X-ray images were characterized by using metrics from regions of interest (ROIs) in the image, which were generated before and after filtering with the Gabor filter bank. In a real-life environment ROIs would be selected manually by the operator using a mouse to delineate the ROI, as shown to Figure $2 \mathrm{~B}$ or automatically as a sliding sub-area moving over the image in a raster manner. In the current work the mean gray levels per ROI (MGR) were obtained from unfiltered and filtered images after they were normalized. MGR's are characterized by the orientation and frequency of the filter that was used and Figure 2B shows an MGR descriptor for the entire ensemble of the regions of interest of a given image. We have called this space 'Uroukov's code'. Figure 2C is an example of this 3D space. When the MGRs are analyzed, it appears that different classes of substances are localized and hence they may be taken as an ensemble that possesses similar characteristics. Searching an image for specific substances can then be carried out on this data set.

\section{Visibility}

Using the I-ImaS concept (Griffiths et al. 2008) the optimal visibility/detection of an object can be obtained by applying feedback control to the X-ray source and/or the detectors. Visibility in a neural and visual context may be considered as the task of obtaining visual information and recognizing a specific portion of it from amongst the rest. In this context, there must be apriori information, i.e. we must know in advance what we are searching for in the image.

In our model, the information about quantity and quality of materials is presented as a 'textural descriptor'. Using these descriptors we define visibility as the ratio, for a given ROI, between enhanced signal and the same ROI without enhancement, $\operatorname{MGR}(f, \theta)^{\text {filtered }} / M$ GR ${ }^{\text {unfiltered, with }}$ parameters frequency (f) and orientation $(\theta)$.

\section{Instruments, Methods and Samples.}

\section{$X$-ray Imaging System}

The imaging system consisted of an X-ray source (XTek, SR125, X-tek Systems) which was located $80 \mathrm{~cm}$ from the detector. The sample was placed immediately in front of the imaging array, (Pax Scan 4030A). The imaging system was calibrated across the range of exposures to be used. These calibration fields enabled all images to be corrected for dark current noise and non-uniformity of response.

During imaging of test packages the incident X-ray flux was monitored using a Keithly ionisation chamber and electrometer (type 3050, Keithley). All images were originally created in '.viv' format and later converted to '.tif' with no compression and 16 bit resolution. The PaxScan4030A acquires images with a resolution of $2304 \times 3200$ pixels with a pixel pitch of $127 \mu \mathrm{m}$. To comply with industry standards of airport baggage scanners, pixels were binned to a pitch of $800 \mu \mathrm{m}$. Binning was performed so as to preserve the original image proportions without biasing the width or height of the image.

\section{Computing Environment}

The algorithms were developed in the Matlab ${ }^{\circledR}$ (Release 2009), The MathWorks Inc. language and were run on a Dell Precision Workstation with $2 x$ Intel $^{\circledR}$ Xeon $^{\circledR}$ Quad Core processors with 12 GB of 
RAM. Typical run times were les then a second, which could be significantly influenced, if implemented on dedicated hardware platform. These algorithms have also been successfully used in a different application (Uroukov, et al. 2014).

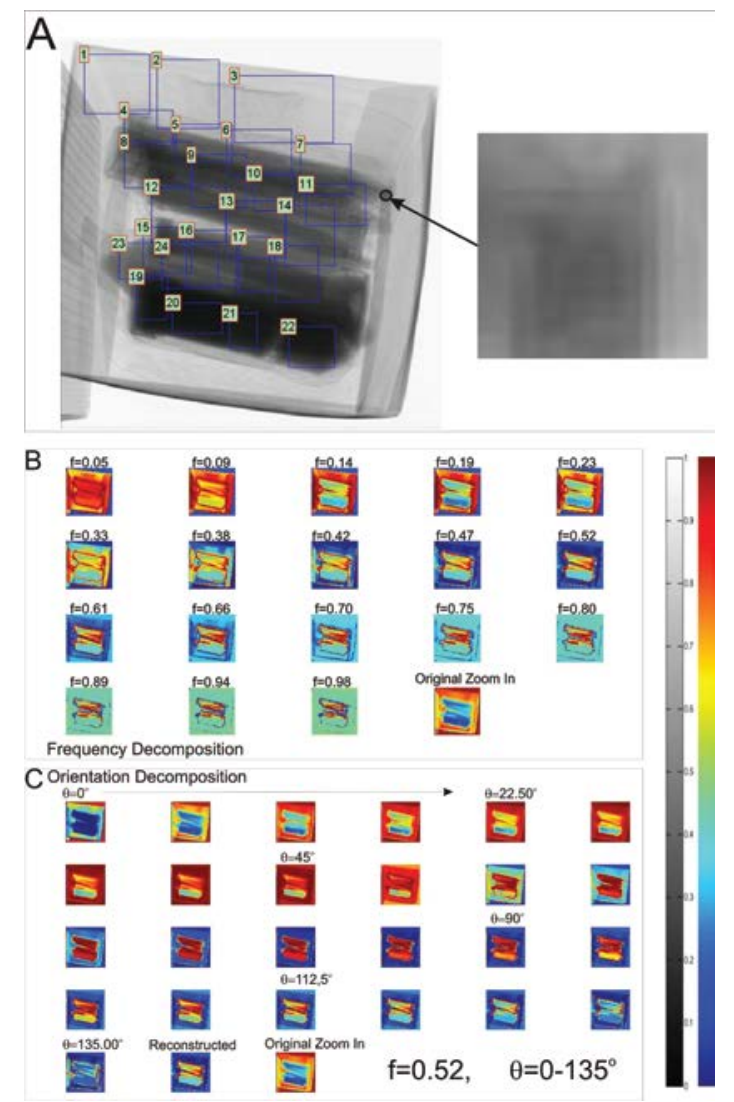

FIG. 3 A X-RAY IMAGE OF BOXED PACKAGE WITH ORGANIC MATERIALS OBTAINED AT $80 \mathrm{KV}$. THE MATERIALS ARE IN TEST TUBES AND BUBBLE WRAP PACKING AND PLACED IN A CARDBOARD BOX. THE ROIS ARE TAGGED WITH NUMBERS

IN THE CORNER AND OUTLINED IN BLUE. HIGHLY MAGNIFIED SUBTLE MIXTURE OF GREY LEVELS AND THEIR SPATIAL ARRANGEMENTS ARE SHOWN FOR A SMALL REGION.

B FREQUENCY DECOMPOSITION PROCESS. AN INTEGRATION OF THE ORIENTATION DECOMPOSITION PROCESS FOR THE RANGE $\theta=0-135^{\circ}$ LEADS TO SERIES OF IMAGES FOR THE

RANGE OF SCANNED FREQUENCIES. THE IMAGES ARE LABELLED WITH THE FREQUENCY. FOR A SELECTED RANGE OF FREQUENCIES AND ORIENTATIONS THE SIGNAL FOR A GIVEN SUBSTANCE (I.E., IN ITS ROI ) VS. THE BACKGROUND (I.E., THE VALUE FROM REST OF THE IMAGE) APPROACHES $100 \%$.

C ORIENTATION DECOMPOSITION PROCESS. THE RECONSTRUCTION OF THE IMAGES IS PERFORMED AT A PARTICULAR FIXED FREQUENCY AND THE ORIENTATION IS CHANGED. ALL ORGANICS ARE WELL DETECTED WHEN THE ORIENTATION IS BETWEEN $\theta=60-90^{\circ}$. THE FILTERED IMAGES AT EVERY ANGULAR STEP ARE SHOWN. AN ACCUMULATIVE RECONSTRUCTION FOR THE ENTIRE RANGE OF ORIENTATIONS IS SHOWN IN A NORMALISED RANGE, LABELLED ‘RECONSTRUCTED’. THE ORIGINAL GREY SCALE IMAGE IS SHOWN FOR COMPARATIVE PURPOSES CONVERTED IN 'JET' COLOUR SCALE.

\section{Sample Preparation}

We were interested in a wide range of low $\mathrm{Z}$ materials such as plastics and organics. Falcon test tubes of 15 and $50 \mathrm{ml}$ were filled with various organic materials including food products, ethanol, and condensed and liquid oils. The tubes were packed with bubble wrap and tape and packed in a cardboard box.

\section{Results}

\section{Image Decomposition}

The test sample box was imaged using X-ray exposure conditions that might be encountered at an airport site. Depending upon the imaging modality available at the airport, multiple views maybe taken but typically only one is used. To test the algorithms, images were acquired from four directions (labelled front, rear, left and right sides). Figure $3 \mathrm{~A}$ is the 'front' image and presents the typical grey scale X-ray image with numbered ROIs outlined in blue. Subtle mixtures of grey levels and their spatial arrangement are difficult to see in X-ray images unless highly magnified. Small regions are shown in Figure 3A. Two image decompositions were performed, one through a range of frequencies and another through different orientations. The frequency decomposition is presented in Figure 3B (in colour map ' $j e t$ ') where the different images are labelled with the frequency of reconstruction. The ability of the filter bank at certain frequencies (e.g., in the frequency band $\mathrm{f}=0.42-0.80$ ) to select particular types of substances can be seen. Figure $3 \mathrm{C}$ shows the orientation decomposition at the frequency where the energy measure, i.e., the MGR, is a maximum. At this frequency, the orientation decomposition demonstrates an ability to select organics in the angular range of $60^{\circ}-80^{\circ}$.

\section{Minimal Resolution Conditions and Opearational Limits}

Airport security scanners do not operate with large area detectors such as the PaxScan system used in these experiments. Instead baggage scanners use line scan technology to acquire data and these conditions were simulated using only strips of data taken from the full 2-D, PaxScan images. Using the large area detector in this way fully simulates the normal operation of a line scan system. In most baggage scanners the pixel pitch is of the order of $800 \mu \mathrm{m}$ thus enough columns of data were chosen to consider how the system would work with 10 to 30 strips of line scanning detectors. This allowed the effect of strip 
width on the operation of the filter bank to be studied. Figure 3 shows the results of these simulations. A 'standard suitcase' was imaged and is shown in 'dual energy' colour-coding mode in Figure 4A. Figure 4B is shown in 'jet' color-coding after decomposition is performed over the entire image. Figures $4 \mathrm{C}, 4 \mathrm{D}$ and $4 \mathrm{E}$ show reconstructions with different sized widths for the line-scan detector simulations. It can be seen that the operation of the filter was still able to recognise material textures down to a strip width of 15 pixels, significantly smaller than current baggage scanners. Most baggage scanners operate the X-ray source at $120-140 \mathrm{kV}$. In our laboratory system the source had a maximum potential of $80 \mathrm{kV}$. This implies a less penetrating beam and hence a more noisy image for the same X-ray tube current but does provide better image contrast. These changes have opposing effects upon detectability and hence it is expected that the overall effect upon the ability to detect objects would not be significant.
A

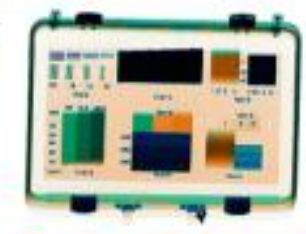

B

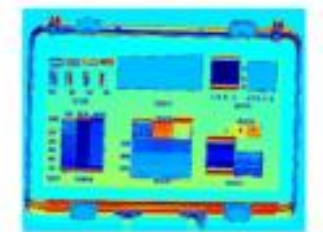

$\mathrm{E}$

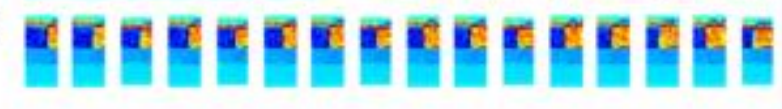

FIG. 4 STEERING ALGORITHM RESULTS ARE PRESENTED. HERE WE SHOW THE EFFECT ON ANALYSIS OVER PORTION OF DATA, ACQUIRED WITH A LINE DETECTOR AS IT IS AT THE AIRPORT SCANNERS. A STANDARD SUITCASE WAS IMAGED (IMAGE A) IN DUAL ENERGY MODE WITH AN AIRPORT SCANNER (3D X-RAY LTD. UK) AND PRESENTED IN

COLOR-CODING AS FOLLOW: ORANGE FOR ORGANIC MATERIALS, GREEN FOR INORGANIC MATERIALS AND BLUE FOR METALS. THE RESULT OF ANALYSIS FOR ENTIRE IMAGE IS SHOWN AS IMAGE B IN 'JET' COLOR-CODING. IMAGES C

AND D SHOWS A VARIATION OF THE SLIT SIZE ON THE

ANALYZED PORTION (ALSO PRESENTED ON E,) OF THE IMAGE AND THEIR RECONSTRUCTIONS. THE SIMULATED SLIT SIZE IS FAR BELOW THE ‘PHYSICAL DEMANDS' (SLIT

WIDTH) OF THE REAL SCANNER, WHICH SHOW A SATISFACTORY RESULT OF THE DETECTION ALGORITHMS. STRIP WIDTH FOR IMAGE C IS 50 PIXELS. STRIP WIDTH FOR IMAGE D IS 25 PIXELS. STRIP WIDTH FOR IMAGE E IS 15 PIXELS.

\section{Model of Visibility for Computing Purposes}

An X-ray image of sample at $80 \mathrm{kV}$ is shown on Figure
$5 \mathrm{~A}$. Figure 5B demonstrates the result of applying the model for various ROI's and shows the computed visibility per ROI. The sample was imaged over a range of X-ray tube currents in order to achieve a range of contrast to noise ratios. As an example, it can be seen that particular materials i.e. ROI 1, are 'visualized' better by the system at a current of $100 \mu \mathrm{A}$ when tube voltage is held at $35 \mathrm{kV}$. It can also be seen that the calculated visibility is not consistent for all ROIs and depends on the filter bank selectivity. The standard deviation is not shown on these results as it is insignificant compared to the mean values.

\section{Visibility and use of 'Uroukov's Code'}

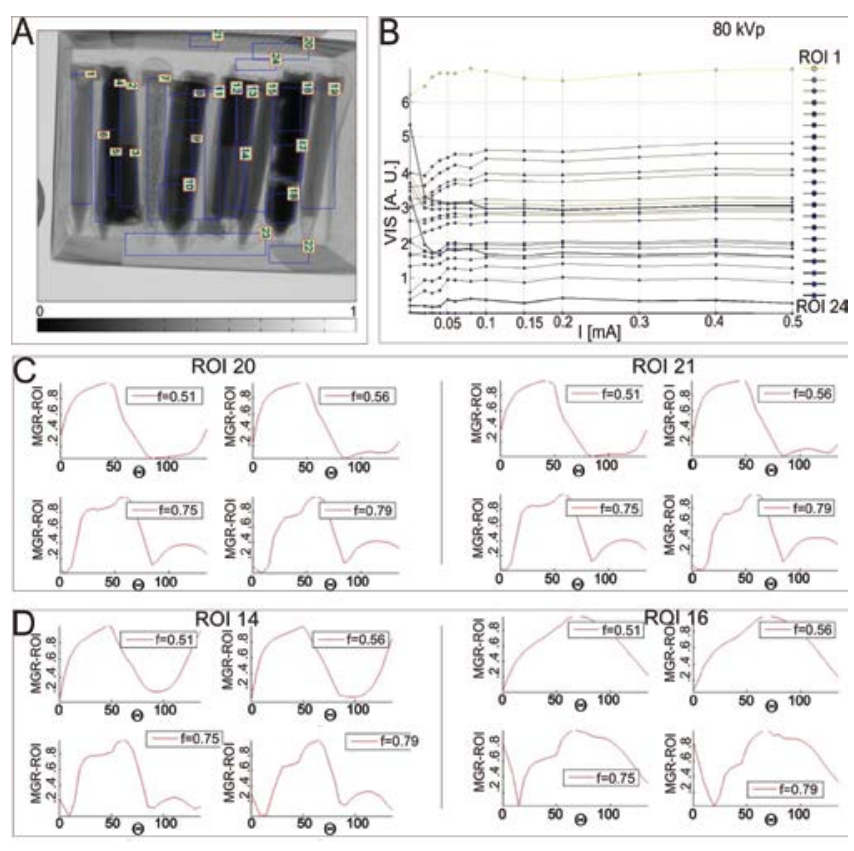

FIG. 5 A X-RAY IMAGE OF THE SAMPLE OBTAINED AT $80 \mathrm{KV}$. B CHANGE IN THE VISIBILITY AS A RESULT OF CHANGE IN THE X-RAY EXPOSURE CONDITIONS (I.E., CHANGE IN X-RAY TUBE CURRENT). THIS WAY WE MAY IMAGE PARTICULAR MATERIALS IN CONDITIONS OF 'MAXIMUM VISIBILITY' AT LOWEST X-RAY DOSE. THE FAMILY OF CURVES (VISIBILITY IN ABSOLUTE UNITS VS. X-RAY TUBE CURRENT IN MA) SHOWS THE RESULTANT VISIBILITY AT FIXED CONDITIONS FOR SELECTED ROIs. PARAMETERS: $F=0.52$, IMAGES INTEGRATED IN THE RANGE OF ORIENTATIONS BETWEEN 60-80 DEGREES. C DEMONSTRATES THE SIMILARITY IN THE DESCRIPTOR FUNCTIONS FOR VERY SIMILAR MATERIALS I.E. ROI20 AND ROI21. SERIES OF GRAPHS PRESENT PARTICULAR ROIS TEXTURAL DESCRIPTORS. EACH DESCRIPTOR IS A PLOT OF THE MGR RESPONSE IN AU V.S. $\theta$ (THE ORIENTATION) IN DEGREES AT GIVEN FREQUENCY.

D DIFFERENT DESCRIPTORS CORRESPOND TO THE DIFFERENT MATERIALS AT ROI 14 AND ROI 16 ARE PRESENTED.N

As described, 'Uroukov's code' space is the integrated signal strength from the ROIs, which include the response of a particular texture to the filter bank for a given set of parameters. To illustrate the response of 
certain materials after passing through a filter bank, we show the integrated signal strength from organic materials with similar X-ray absorption characteristics (Figure 5C, ROI-20 and ROI-21) and different absorption characteristics (Figure 5D, ROI-14 and ROI16) for a full set of parameters of the filter bank. These materials were: ROI 20 and ROI 21 cardboard; ROI 14 lard and ROI 16 melted chocolate. It can be seen that similar materials have similar descriptors and these are plotted for a range of frequencies (i.e. $\mathrm{f}=0.05-0.98$ ) and labeled appropriately.

\section{An Example of Using the Model to Enhance Detection - Tobacco Detection}

A particularly challenging example in conventional Xray scanning is the identification of tobacco. A test object was constructed of different layers:

- Layer 1 Organic materials comprising various foods.
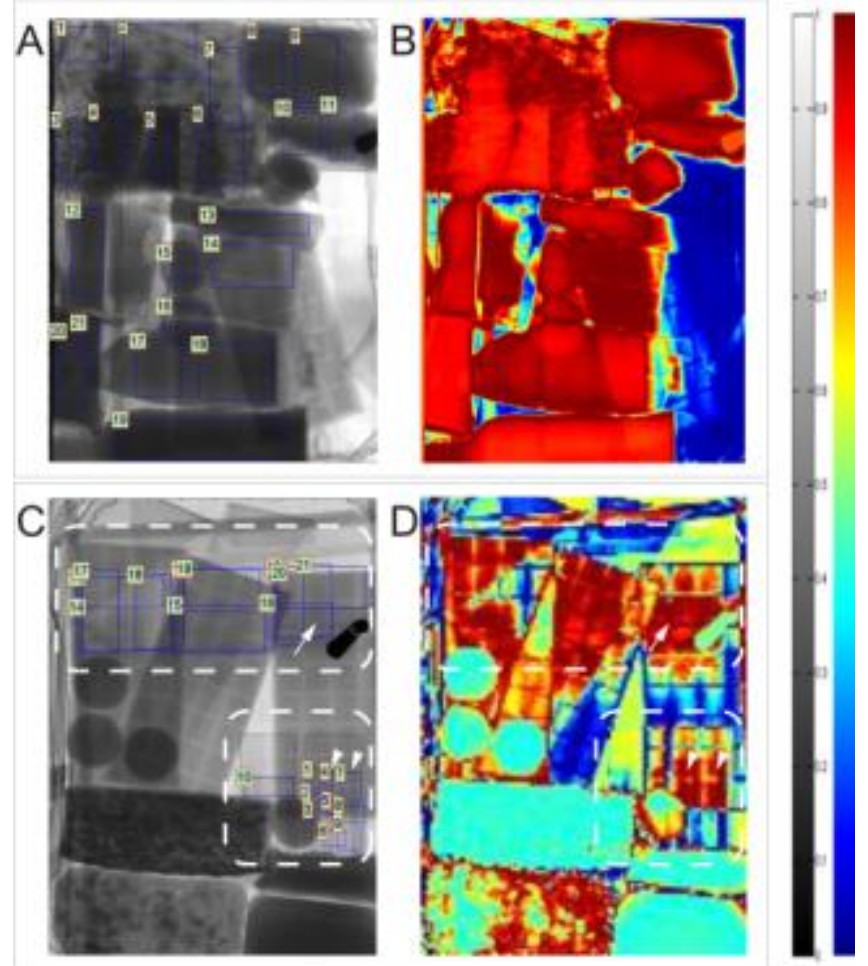

FIG. 6 X-RAY IMAGE OF ORGANIC CONTENT PACKED IN A RUCKSACK WITH THE COMPUTED RESULTS FOR THE DETECTION OF ALL ORGANICS AS WELL AS THE SPECIFIC DETECTION OF TOBACCO OBTAINED AT 80KV. IMAGE AREAS WHERE TOBACCO WAS HIDDEN ARE INDICATED WITH THE WHITE BROKEN LINE. THE 'ARROW' INDICATES ONE OF THE HIDDEN LOOSE TOBACCO PACKAGES AND THE

'ARROWHEADS' INDICATE HIDDEN BOXED CIGARETTES.

A, C GREY SCALE X-RAY IMAGES OF COMBINED ORGANIC MATERIALS INCLUDING FOOD, LIQUIDS AND GELS B - DETECTION OF THE ENTIRE ORGANIC CONTENT

D - DEMONSTRATION OF TOBACCO DETECTION (LOOSE AND CIGARETTES).
- Layer 2 Organic materials comprising cosmetic and liquid-gel substances.

- Layer 3 Tobacco products.

As it is very complicated to distinguish tobacco from paper and other dry products with high fibre and cellulose content based on their X-ray absorption, most of the current imaging systems fail to distinguish these products from drugs such as marihuana if they are not spectroscopically analysed. Figures 6A and 6C present grey scale $\mathrm{X}$-ray images with numbered ROIs. Figure $6 \mathrm{~A}$ shows an imaged bag with organic content without tobacco while Figure 6C shows a mixture of organics layers with a layer of tobacco in between.

Figure 6B shows the detection of the entire organic content and Figure 6D demonstrates detection of tobacco products hidden between foods layers. In Figure 6C, the tobacco (in the form of loose, cut tobacco and cigarettes in boxes) is hardly visible (highlighted with white punctured line) as the image is dominated by more the absorbing chocolate. However, in Figure 6D the processed image clearly shows the presence of tobacco in its different forms.

\section{Discussion and Conclusions}

From the work carried out in this preliminary study we have been able to show that there is a technological solution for the non-trivial security issue of characterising materials during $\mathrm{X}$-ray scanning. Combining adaptable X-ray scanning technology (e.g., I-ImaS (Griffiths et al, 2008)) with signatures of materials obtained 'on-the-fly' would allow imaging parameters to be adjusted in real-time to optimise detection. To realise this we implemented a textural model for the characterisation of imaged materials.

Our work has been aimed at the detection of security related energetic materials. Generally, materials with similar X-ray absorption are recorded with similar grey levels in the image and hence current technology uses dual-energy X-ray scanning to help classify materials. However, many organic materials mimic the dual-energy appearance of explosives. Thus we have looked at the use of textural analysis on organic materials as a way to aid classification.

We imaged and analysed a range of materials that might mimic liquid and plastic energetic materials. Evaluated samples show that is possible to distinguish between materials due to their differences in the texture pattern despite having very similar absorption coefficients. 
In order that this concept can be used to control an adaptive X-ray scanner it is necessary to make the preliminary regional characterisation on strips of data taken from line scanning technology. In this work it has been shown that strips of acquired data, consistent with current baggage scanner technology, are adequate to characterise materials and allow on-the-fly adaptation of the scanning system. In this proof of concept work we have used high level languages and a desk-top PC to run the algorithms. In a real-world scenario where the processing would be required to produce real-time decisions, the computing environment would need to be carefully considered. This would probably require embedded firmware to achieve the timescales required.

Adopting this real-time, adaptive approach to baggage scanning would mean changes to current systems. These changes are technological in nature (generation of feedback signals, X-ray generator controls, etc) and have not been the concern of this paper. However, it is clear that operated in the way described in this paper the opportunity exists to use 'quick look' data to make the appropriate decisions that would allow adaptation of exposure factors on a bag-by-bag basis to provide improved images.

\section{ACKNOWLEDGMENT}

This project was funded under the Innovative Research Call in Explosives and Weapons Detection (2008) initiative, a cross-government programme sponsored by a number of government departments and agencies under the CONTEST strategy.

\section{REFERENCES}

Abidi B., Liang J. Mitckes M., Abidi M., 2004. Improving the detection of low-density weapons in x-ray luggage scans using image enhancement and novel scene-decluttering Techniques, Journal of Electronic Imaging 13(3), 523-538 . AL-Hinnawi A. R. and Daear M., 2012. Image Texture Descriptors to Quantify Bilateral Filter on Low Dose Computerized Tomography. International Journal of Signal Processing. Image Processing and Pattern Recognition, 5(3), 123-136.

Azzopardi George, Petkov Nicolai, 2012. A CORF computational model of a simple cell that relies on LGN input outperforms the Gabor function model. Biol Cybern., 106, 177-189.

Campbell F.W. and Kulikowski J.J., 1966. Orientational sensitivity of human visual system. Journal of Physiology, 187, 437-455.

Clausi D. A., Jernigan M.E., 2000. Designing Gabor filters for optimal texture separability. Pattern Recognition, 33, 1835-1849.

Coggins J.M. and Jain A.K., 1985. A spatial filtering approach to texture analysis. Pattern Recognition Letters, 3(3), 195-203.

Daugman J.G., 1980. Two-dimensional spectral analysis of cortical receptive filed profiles. Vision Research, 20, 847856.

Daugman J.G., 1985. Uncertainty relation for resolution in space, spatial frequency, and orientation optimized by two-dimensional visual cortex filters. J. Opt. Soc. Am. A.2(7), 1160-1169.

Farhad Ghelmansarai, PerkinElmer Holdings Inc., 2012, US, Patent nr. 20120033793, Adaptive frame scanning scheme for pulsed X-ray. IPC8 Class: AG21K510FI USPC Class: 378146, Patent application number: 20120033793.

Franzel T., Schmidt U, Roth S., 2012. Object Detection in Multi-view X-Ray Images.Pattern Recognition, Lecture Notes in Computer Science Volume 7476, pp 144-154.

Gesick R, Sartic C, Chih-Cheng Hung, 2009. Automatic Image Analysis Process for the Detection of Concealed Weapons, CSIIRW ,Proceedings of the 5th Annual Workshop on Cyber Security and Information Intelligence Research: Cyber Security and Information Intelligence Challenges and Strategies, Article 20.

Griffiths J. A., Metaxas M. G., Pani S., Schulerud H., Esbrand C., Royle G. J., Price B., RokvicT., LongoR., AsimidisA., Bletsas E., Cavouras D., Fant A., Gasiorek P., Georgiou H., Hall G., JonesJ., Leaver J., LiG., Machin D., ManthosN., Matheson J., Noy M.,OstbyJ.M., Psomadellis F., van der SteltP.F., TheodoridisS., TriantisF., TurchettaR., VenanziC., SpellerR.D., 2008. Preliminary images from an adaptive imaging system, Physica Medica, 24(2), 117-21.

Huda W, Sajewicz. A. M., Ogden K.M., Dance D.R., 2003. Experimental investigation of the dose and image quality characteristics of a digital mammography imaging system. Med. Phys., 30(3), 442-448.

Jain A. K. and Farrokhnia F., 1991. Unsupervised texture segmentation using Gabor Filters. Pattern Recognition, 24(12), 1167-1186. 
Jain A.K., Ratha N.K., and Lakshmanan S., 1997. Object Detection Using Gabor Filters, Pattern Recognition 30(2), 295-309.

Jones, Judson P., Palmer Larry A., 1987. An evaluation of the two-dimentional Gabor filter model of simple receptive field in cat striate cortex. Journal of Neurophysiology, $58(6)$.

Julesz B., 1962. Visual pattern discrimination. IRE Trans. on Information Technology, IT-8, 84-92.

Julesz B., 1981. A theory of preattentive texture discrimination based on first order statistics of textons. Biol. Cybernetics, 41, 131-178.

Julesz Bela, 2011. Preattentive human vision: link between neurophysiology and psychophysics. Chapter 14, AT \& T Bell Labs, Published in: Comprehensive Physiology. DOI: 10.1002/cphy.cp010514

Longo R., Asimidis A., Cavouras D., Esbrand C., Fant A., Gasiorek P., Georgiou H., Hall G., Jones J., Leaver J. Li G., Griffiths J., Machin D., Manthos N., Metaxas M., Noy M., Østby J. M., Psomadellis F., Rokvic T., Royle G., Schulerud H., Speller R., van der Stelt P.F., Theodoridis S., Triantis F., Turchetta R., Venanzi C., A scanning system for intelligent imaging: I-ImaS, Proc. SPIE 6510, Medical Imaging 2007: Physics of Medical Imaging, 65100M (March 13, 2007); doi:10.1117/12.708457, 2007.

Lin Hong, Yifei Wan, and Anil Jain, Fingerprint image enhancement: Algorithm and performance evaluation. IEEE Transaction on pattern analysis and machine intelligence, Vol. 20, No. 8,1998

Marcelja S., 1980. Mathematical description of the responses of simple cortical cells. J. Opt. Soc. Am., 70(11), 1297-1300. Martin C.J., Sutton D.G., Sharp P.F., 1999. Balancing patient dose and image quality. Applied Radiation and Isotopes, 50, 1-19.

Mery D., Riffo V., Zuccar I., Pieringer C., 2013. Automated X-Ray Object Recognition Using an Efficient Search Algorithm in Multiple Views, Proceeding CVPRW '13 Proceedings of the 2013 IEEE Conference on Computer Vision and Pattern Recognition Workshops Pages 368374.

Mohamed S. S., Abdel-galil T. K., Salama M. M. A., Elsaadany E. F., and Kamel M., Fenster A., Downey D. B., and Rizkalla K., 2013. Prostate cancer diagnosis based on Gabor filter texture segmentation of ultrasound image.
CCECE 2003 - CCGEI 2003, Montreal, May/mai 2003 07803-7781-8/03/\$17.00 02003.

Petkov N., Kruizinga P., 1997. Computational models of visual neurons specialised in the detection of periodic and aperiodic oriented visual stimuli: bar and grating cells, Biol. Cybern., 76, 83-96.

Rao A.R. and Lohse G.L., 1993. Identifying high level features in texture perception. GVGIP: Graphical Models and Image Processing, 55 (3), 218-233.

Ulaby F.T., KouyateF., BriscoB., and WilliamsT.H.L., 1986. Textural information in SAR images.IEEE Trans. Geosci. Remote Sensing.GE 24, 235-245.

Uroukov I.S. Speller R. and Olivo A., 2014. Image Enhancement of X-ray Phase Contrast Images of Micro Objects. Signal Processing Research, Vol. 3,pp. 24-30,2014. Yunhong Wang, Tieniu Tan, Jain A. K., 2003. Combining Face and Iris Biometrics for Identity Verification, J. Kittler and M.S. Nixon (Eds.): AVBPA 2003, LNCS 2688, pp. 805-813, 2003. Springer-Verlag Berlin Heidelberg 2003.

Yu Shi and Xian-Da Zhang, 2001. A Gabor atom network for signal classification with Application in Radar Target Recognition, IEEE Trans. On signal processing. Vol. 49, No. 12.

Zhiyu Chen, Yue Zheng, Abidi B. R., 2005. A Combinational Approach to the Fusion, De-noising and Enhancement of Dual- Energy X-Ray Luggage Images. Proceedings of the IEEE Computer Society Conference on Computer Vision and Pattern Recognition (CVPR'05), article number 1565297.

Willson P.D. 1997. Apparatus and method for automatic recognition of concealed objects using multiple energy computed tomography, Patent Nr. WO 1997018462 A1.

WoodellG. Zia-ur Rahman Jobson D.J, Hines G., 2004. Enhanced images for checked and carry-on baggage and cargo screening, Proc. SPIE 5403, Sensors, and Command, Control, Communications, and Intelligence (C3I) Technologies for Homeland Security and Homeland Defense III, 582.

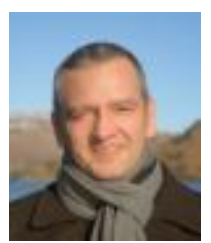

Ivan S. Uroukov (Dipl. Eng. Physicist, MSc, $\mathrm{PhD}$ ) is a graduate in Condensed Matter Physics from the Bulgarian Academy of Sciences, Sofia, Bulgaria.He has worked and published in semiconductor physics, 
biophysical imaging, image computing and biological information processing topics.He has investigated the use of these approaches for examining material science aspects of biomedical and security imaging. He is currently engaged in applying signal and image computing with psychophysical approach to ultra-low contrast imaging techniques, such as electron microscopy of unstained, frozen, biological specimens and similar imaging modalities. Healso has interests in biologically inspired signal and information processing. Dr Uroukov is a member of Society for Neuroscience, USA, 2008.

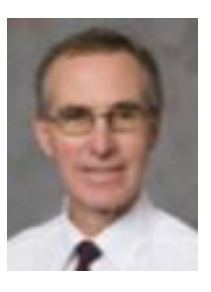

Robert Speller is the Joel Professor of Physics Applied to Medicine at University College London. He is Head of the Radiation Physics Group. A group of $\sim 30$ members with interests in scattered radiation fields, $X-$ ray diffraction, phase contrast imaging, radioisotope mapping,heavy charged particle radiotherapy and tomographic techniques applied to a range of problems covering medicine, security and industry. He holds patents in a range of imaging techniques including phase contrast imaging and is a Fellow of the Royal College of Radiologists. 